\title{
New insights in anterior cruciate ligament morphology: implications for anterior cruciate ligament reconstruction surgeries
}

\author{
Rekha Lalwani $^{1}$, Rohit Srivastava ${ }^{2}$, Sheetal Kotgirwar ${ }^{1}$, Sunita A. Athavale ${ }^{1}$ \\ ${ }^{1}$ Department of Anatomy, All India Institute of Medical Sciences, Bhopal, Madhya Pradesh, ${ }^{2}$ Intern, All India Institute of Medical Sciences, Bhopal, \\ Madhya Pradesh, India
}

\begin{abstract}
The frequency of injury of anterior cruciate ligament (ACL), the importance of anatomy in surgical repair and potential of the injured ACL to predispose to osteoarthritis necessitates the need for understanding its precise anatomy. Available studies have focussed on tunnel positioning and hence attachment site of the ligament. Few studies which have focussed on morphology reflect ambiguities in size, fibre bundle, number and disposition. Since a near anatomical repair of the ACL is the treatment of choice, the present study was planned to describe the morphology of ACL. Twenty-two ACL (11 right and 11 left) isolated from knee joints of collection of adult embalmed lower limbs were utilised for the study. The ACL morphology and morphometry were studied for footprints and fibre bundles. Three distinct bundles of differing lengths constitute a spiral ACL complex. These bundles are positioned as- intermediate with posteromedial and anterolateral flanking in respective positions. The tibial and femoral footprints are paw-shaped and oval respectively. Tibial footprints are approximately 2.5 times larger in area than the femoral footprints. The unique morphology of ACL vis a vis its spiralization, and its mechanical advantage of in terminal extension and conjunct rotation of knee (which are peculiarly human trait) are discussed. It is recommended to utilize the concept of spiralization and differing bundle length in ACL surgeries to achieve the favourable clinical outcome.
\end{abstract}

Key words: Anterior cruciate ligament, Mechanics, Knee joint, Osteoarthritis

Received May 15, 2020; Revised August 11, 2020; Accepted August 13, 2020

\section{Introduction}

Anterior cruciate ligament (ACL) is attached to the anterior inter-condylar area of the tibia, just anterior and slightly lateral to the medial tibial eminence, partly blending with

\author{
Corresponding author: \\ Sunita A. Athavale (1) \\ Department of Anatomy, All India Institute of Medical Sciences, \\ Bhopal, Madhya Pradesh 462020, India \\ E-mail:sunita.anatomy@aiimsbhopal.edu.in
}

Rohit Srivastava's current affiliation: Department of General Surgery, NSCB Medical college and Hospital Jabalpur, Madhya Pradesh, India the anterior horn of the lateral meniscus. It ascends posterolaterally, twisting on itself and fanning out to attach high on the posteromedial aspect of the lateral femoral condyle [1]. Standard texts of anatomy describe ACL being formed of two, or possibly three, functional bundles that are not apparent to the naked eye but can be demonstrated by microdissection techniques [2].

ACL is one of the most commonly injured ligaments in the knee injuries and has long-term effects on the musculoskeletal system, causing early osteoarthritis. This is of particular concern in children and adolescents as competitive sports participation increases [3] resulting in the rise in ACL reconstruction (ACL-R).

Current trends in ACL-R have been towards a more ana- 
tomic reconstruction that restores the normal size and location of the ACL insertions. It is increasingly been recognized that the success of ACL-R hinges extensively on complying with precise anatomy of ACL. However despite recent studies on ACL morphology ambiguities persist regarding the location of the femoral attachment of postero-lateral bundle [4], sizes of the ACL insertion sites [5] and the number of anatomic bundles that constitute the ACL [6].

Studies of ACL are ambiguous regarding the number of bundles and their respective disposition within ACL. While some studies have reported two bundles namely anteromedial and posterolateral [6-8] few authors have described three bundles namely anteromedial, posterolateral and intermediate [6]. Tan et al. [9] did not find any distinct fibre bundles. Also studies till date have neither described relative fibre disposition within individual bundles and nor have described the footprints in reference to its shape and area [10]. The recently developed two bundle ACL-R surgery also lacks sufficient anatomic background. The existing ambiguities/ disparities in knowledge and understanding morphology and morphometry of ACL have prompted us to conduct this study.

The present study was planned to better understand the anatomy of the ACL to create baseline data for the Indian population. Findings of the present study are expected to aid improved outcomes in ACL-R surgeries.

\section{Materials and Methods}

Twenty-two adult knee joints (11 left and 11 right) obtained from embalmed donated cadavers were utilized for this study after approval from the Institutional Human Ethics Committee (IHEC) of our Institute Letter no IHECLOP/2015/STS0064. All the limbs were fixed with knee joints in extended positions. The sex of the knee joints utilized could not be ascertained as the disarticulated lower limbs available in the collection of Department of Anatomy were utilized for the study. Knee joints with any evidence of congenital or acquired knee pathology affecting ACL complex were excluded from this study.

The knee joint was exposed from the anterior aspect; the skin over the knee joint was reflected by giving an 'I' shaped incision. The incision extended vertically from the middle of the thigh to the middle of the leg. The structures were cleaned; also the connective tissue and the fat were cleared off. The quadriceps tendon was cut immediately proximal to the patella and the incisions were carried downwards towards the tibial condyles passing $2-3 \mathrm{cms}$ on either side of ligament patellae. Patellae were turned downwards to expose the cavity of the knee joint.

The ACL was resected by cutting the tibial and femoral attachments. The synovial membrane of the ACL was removed and the margins were marked.

\section{To document the footprints of anterior cruciate ligament}

The ACL was detached from its tibial and femoral attachments. The tibial and femoral footprints margins were circumscribed by a permanent marker. These footprints were then translated on a piece of the glass plate by apposing each footprint onto the glass plate and creating a replica of the footprint on the surface of the glass plate. These were then further translated and charted on a graph paper to calculate the surface area of each footprint.

The volumetry of each ACL was done by the water displacement method. For this, the ACL was immersed in a glass beaker completely filled to the brim with water. ACL was immersed in this beaker. The water displaced was collected in a container kept underneath the beaker. The displaced water was then measured for its volume in a highly calibrated syringe to measure the exact quantity of water displaced.

Length of ACL was measured from the most anterior point of tibial footprint to the most anterior point of femoral footprint with the help of scale and thread.

The synovial membrane was carefully removed. Different fibre bundles along with their relative positions were identi-
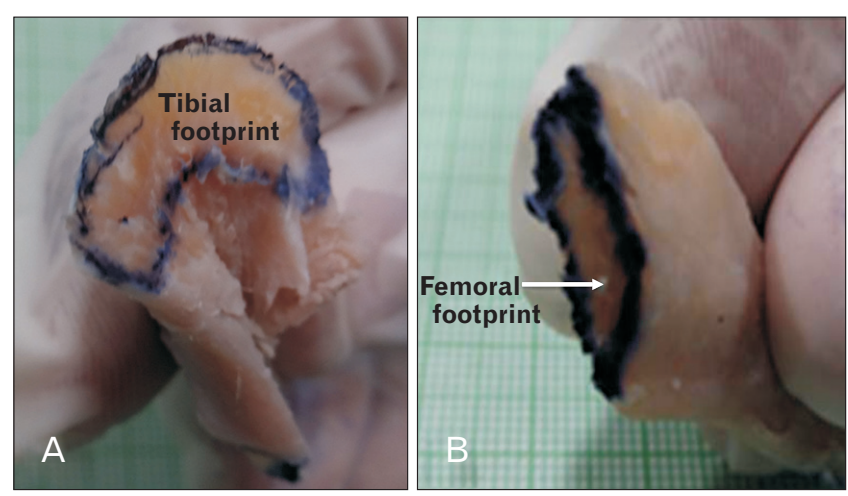

Fig. 1. Shows the footprints of ACL. (A) Shows the morphology of paw-shaped tibial footprints. (B) Shows oval, elongated and mediolaterally flattened femoral footprints. ACL, anterior cruciate ligament. 
fied under a magnifying glass. Disposition of fibres in individual fibre bundles was also noted.

After identification of different fibre bundles, the femoral and tibial footprints charted earlier were revisited and relative position of different fibre bundles was marked in these footprints. The fibre bundles were separated and their morphology and morphometry were also documented.

Statistical analysis of the morphometric measurements for descriptive statistics was done on IBM SPSS Statistics ver. 21.0 software (IBM Co., Armonk, NY, USA).

\section{Results}

The following observations were recorded from the ACL isolated from the cadaveric knee.

The tibial footprints were larger than femoral footprints. They were mostly in the shape of paw; sometimes quadrangular or irregular. Femoral footprints were elongated, oval and mediolaterally flattened and were mostly constant in shape (Fig. 1).

Table 1 shows descriptive statistics of surface areas of tibial and femoral footprints.

Table 1. Descriptive statistics showing the surface area of tibial and femoral footprints of ACL on the right and left side

\begin{tabular}{cccccc}
\hline & \multicolumn{2}{c}{ Tibia } & & \multicolumn{2}{c}{ Femur } \\
\cline { 2 - 3 } \cline { 5 - 6 } & Right & Left & & Right & Left \\
\hline Mean & 105.25 & 114.00 & & 46.25 & 41.11 \\
(range) & $(84.00-131.00)$ & $(56.00-230.00)$ & $(26.00-67.00)$ & $(24.00-62.00)$ \\
$\begin{array}{c}\text { Standard } \\
\text { deviation }\end{array}$ & 13.90 & 61.59 & & 14.96 & 11.00 \\
\hline
\end{tabular}

All values are in $\mathrm{mm}^{2}$.

ACL, anterior cruciate ligament.
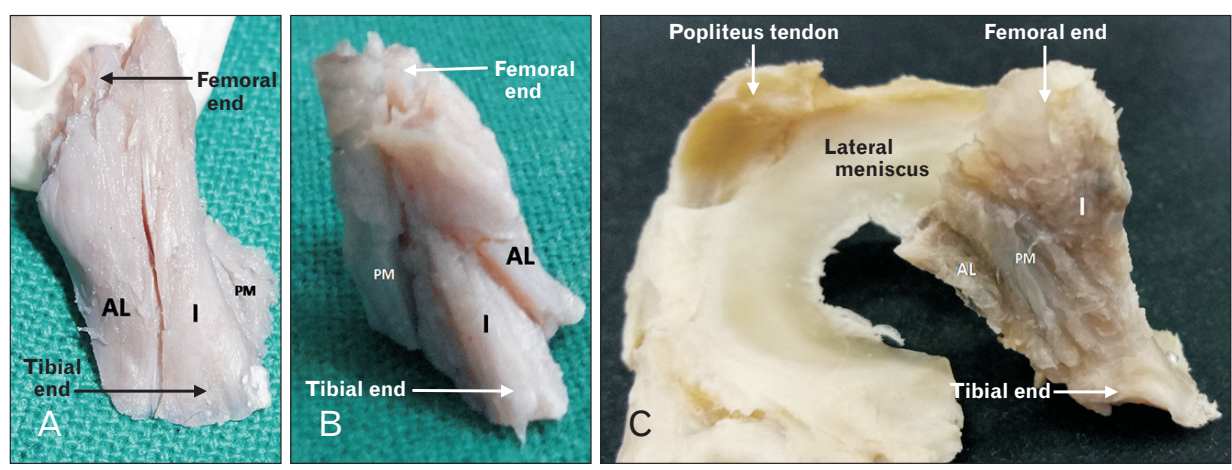

Fig. 2. (A-C) Shows the three bundles of ACL and their relative positioning. Shows the presence of three distinct fibre bundles and the relative positioning of the different fibre bundles as seen in the (A) right and (B) left ACL. C shows ACL from posterior aspect; three bundles can be seen with fibres of posteromedial bundle spiralling posterior to the intermediate bundle. ACL, anterior cruciate ligament; AL, anterolateral bundle; I, intermediate bundle; PM, posteromedial bundle.
The tibial footprints showed more variability as compared to femoral. The difference on two sides was statistically not significant $(P=0.673$-tibial footprints; $P=0.229$-femoral footprints).

Table 2 shows descriptive statistics of ACL length of right and left sides. The length varied greatly across individuals. However, the difference between the two sides was statistically not significant $(P=0.749)$.

After removal of overlying synovium, three distinct fibre bundles could be observed in the entire set of specimen studied.

\section{Intermediate bundle}

The centrally placed bundle was the largest and occupied an intermediate position. Hence was named intermediate by the authors. It was flanked by two more bundles which were positioned anterolaterally (anterolateral bundle) and posteromedially (posteromedial bundle) clasping the intermediate bundle forming a spiral complex (Fig. 2).

The intermediate bundle had an attachment in the central region of the tibial footprint. The fibres of the bundles spiralled about the middle of ACL and than narrowed and flat-

Table 2. Descriptive statistics showing the length of ACL on the right and left side

\begin{tabular}{lcc}
\hline & Right & Left \\
\hline Mean (range) & $30.79(25.17-43.52)$ & $29.91(14.20-40.79)$ \\
Standard deviation & 5.35 & 7.61 \\
\hline
\end{tabular}

All values are in $\mathrm{mm}$.

ACL, anterior cruciate ligament. tened mediolaterally near the femoral footprint (Fig. 3). 


\section{Anterolateral bundle}

The anterolateral bundle was attached on tibia on the lateral part of the tibial footprint. The fibres ran a straight course towards the femur and clasped the intermediate bundle from the anterolateral aspect. It had a mediolaterally flattened attachment onto the femur.

\section{Posteromedial bundle}

The posteromedial bundle was smaller and shorter than the intermediate and anterolateral bundle. It was attached towards the medial and relatively posterior part of the tibial footprint. The fibres ran a straight course towards the femur,

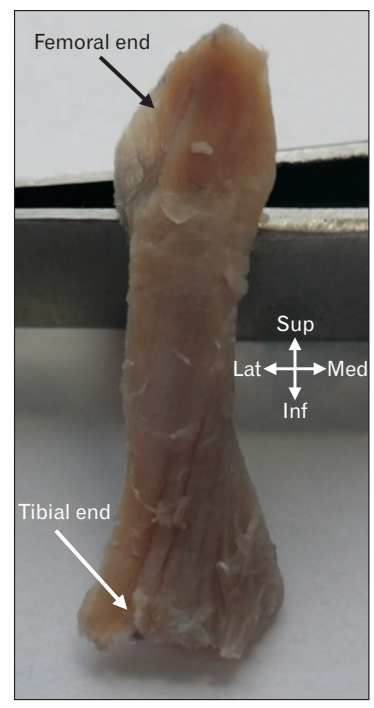

Fig. 3. Shows the medially spiralized fibre arrangement of the intermediate bundle of the right side. Inf, inferior; Lat, lateral; Med, medial; Sup, superior. clasping the intermediate bundle posteromedially. The attachment on femur varied in position with respect to that of the intermediate bundle (Fig. 4).

In two cases (1 right and 1 left) the posteromedial bundle was very small. In these cases, some fibres of anterolateral bundle crossed over from lateral to medial side of the intermediate bundle and got attached to the medial side of the femoral attachment of the intermediate bundle (Fig. 5). The relative positions of fibre bundles in the femoral footprint were classified into four types as shown in Fig. 4.

The three bundles were arranged in such a manner that

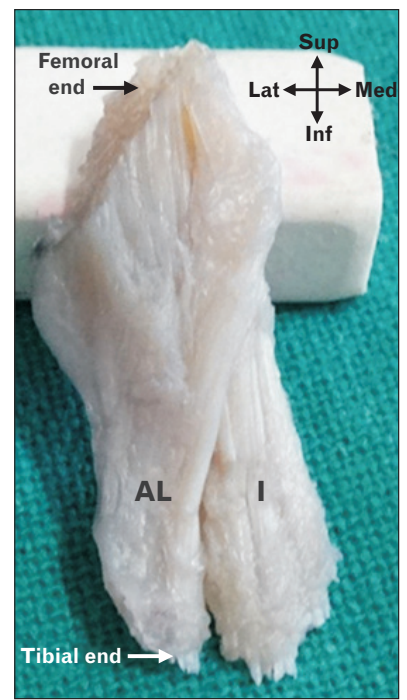

Fig. 5. Showing fanning fibres of AL crossing over from lateral to medial side of the I on the right side. Posteromedial bundle of this ACL was very small and not seen in this view. AL, anterolateral bundle; ACL, anterior cruciate ligament; I, intermediate bundle; Inf, inferior; Med, medial; Lat, lateral; Sup, superior.

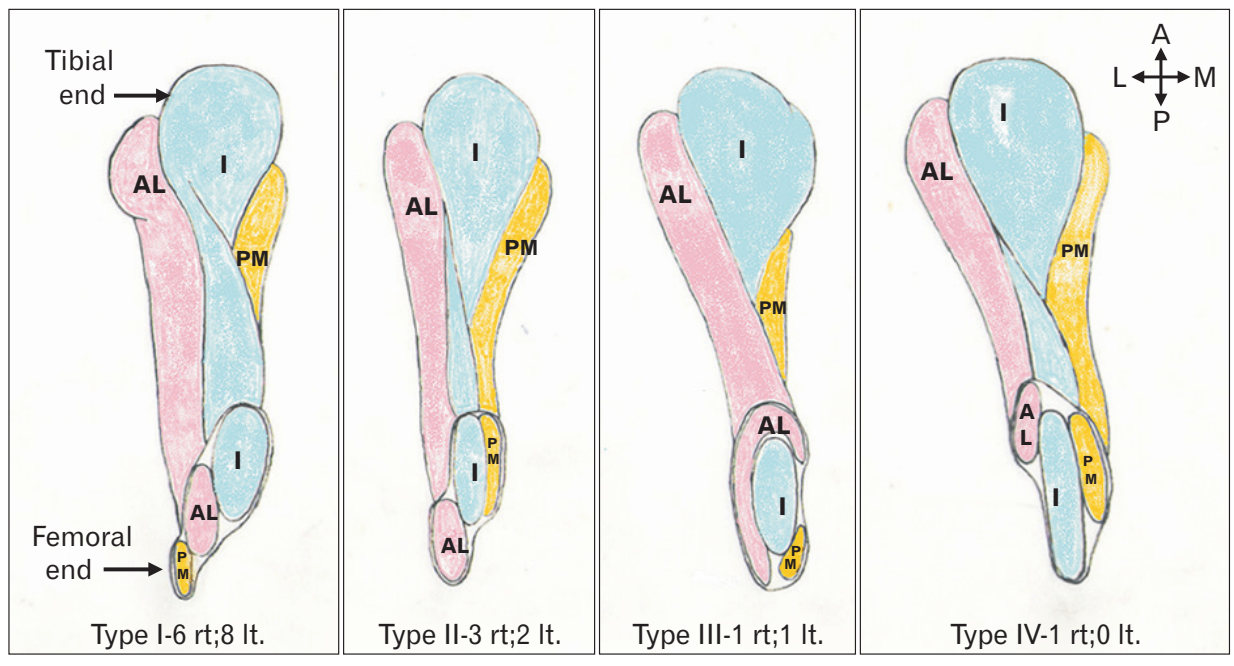

Type I-6 rt;8 It.
Type II-3 rt;2 lt.
Fig. 4. Shows the schematic diagram of ACL as visualized from the superior aspect to see the femoral footprints. The different arrangements of fibre bundles based on their relative position in ACL femoral footprints are seen. A, anterior; ACL, anterior cruciate ligament; AL, anterolateral bundle; I, intermediate bundle; L, lateral; M, medial; P, posterior; PM, posteromedial bundle. 
created a spiral complex with the intermediate bundle (with twisted fibres) was clasped by anterolateral and posteromedial bundles spirally (Fig. 2A, B).

Table 3 shows the volumetric analysis of ACL as observed in the study. The difference between the two sides was statistically not significant $(P=0.721)$.

Table 4 shows the lengths of the individual fibre bundle of ACL. It was observed that the three bundles had different lengths; intermediate bundle was longest and the posteromedial was the shortest.

\section{Discussion}

The frequency of injury of ACL, the importance of anatomy in surgical repair and the potential of injured ACL to predispose to osteoarthritis necessitates the need for understanding the anatomy of ACL. The findings of the present study regarding the tibial and femoral footprints concur with Oka et al. [11] who have described the shape of the tibial footprint as 'duck foot region' and Petersen and Tillmann [12] who have described femoral footprint as an oval. Seibold et al. [13] have reported that the femoral footprint was significantly larger in the area in men as compared to women and left knees as compared to the right. No significant right-left difference was observed in any of the study parameters in the present study.

The mean ACL length was reported to be $44 \mathrm{~mm}$ by Harvey et al. [14] but lower values $(30 \mathrm{~mm})$ have been recorded in the present study.

Tan et al. [9] in their study on Chinese and Singaporean

Table 3. Descriptive statistics showing the volume of ACL on the right and left side

\begin{tabular}{lcc}
\hline & Right & Left \\
\hline \multicolumn{1}{c}{ Mean (range) } & $1.13(0.60-2.00)$ & $1.19(0.50-2.20)$ \\
Standard deviation & 0.41 & 0.52 \\
\hline All values are in ml. & & \\
ACL, anterior cruciate ligament.
\end{tabular}

population have concluded that ACL was shorter, narrower and more vertical in this population. The difference in findings as observed by Tan et al. [9], and in the present study might be due to different landmarks used for morphometric analysis or due to racial differences.

There is a lot of ambiguity regarding the number and nomenclature of the fibre bundles of ACL. Most authors agree that the ACL can be divided into functional and anatomically distinct bundles- anterolateral and posteromedial bundles. Some authors have reported an additional third intermediate bundle [2, 6]. However, Tan et al. [9] have reported that they did not find any distinct anatomical bundles in the ACL and concluded that these bundles are more functional rather than anatomical. Siebold et al. [15] and Smigielski et al. [16] have proposed a ribbon-like flat ACL concept devoid of any bundles. However, the present study has unambiguously identified and documented the presence of three distinct bundles of ACL which differ in position, length and fibre orientation.

\section{Relative positioning and nomenclature of anterior cruciate ligament bundles}

Our findings conform to the findings of Ferretti et al. [6], who have also documented the presence of three anatomical bundles by gross anatomy and histology. The nomenclature of these bundles described by most authors and standard textbooks of anatomy vis a vis as observed in the present study differs. Authors who have described two bundles have named them anteromedial and posterolateral. Documentation of the third bundle is bound to change the current nomenclature. These studies seemed to have overlooked a small but constant bundle distinctly separate from the other two; the posteromedial bundle.

\section{The posteromedial bundle}

This bundle is medial most, placed relatively posterior in tibial footprint and clasps the intermediate bundle from pos-

Table 4. Showing length of different bundles of ACL

\begin{tabular}{|c|c|c|c|c|c|c|}
\hline & \multicolumn{3}{|c|}{ Bundle length: right ACL } & \multicolumn{3}{|c|}{ Bundle length: left ACL } \\
\hline & Anterolateral & Intermediate & Posteromedial & Anterolateral & Intermediate & Posteromedial \\
\hline \multirow[t]{2}{*}{ Mean (range) } & 27.54 & 31.34 & 23.04 & 30.25 & 32.03 & 24.02 \\
\hline & $(19.29-37.89)$ & $(23.54-37.71)$ & $(18.72-29.78)$ & $(22.22-40.58)$ & $(20.25-43.28)$ & $(13.70-35.24)$ \\
\hline Standard deviation & 5.83 & 4.66 & 4.35 & 6.34 & 7.58 & 6.82 \\
\hline
\end{tabular}

All values are in $\mathrm{mm}$.

ACL, anterior cruciate ligament.

Difference between right and left was statistically not significant. 
terior aspect (Fig. 2). This has hitherto not been described in the available literature to the best of our knowledge.

\section{The intermediate bundle}

This conforms to the anteromedial bundle described by other authors, is central in position, and is placed most anterior in tibial footprint (Fig. 2).

\section{The anterolateral bundle}

The third bundle described as anterolateral in the present study is the lateral most, placed relatively anterior as compared to the posteromedial bundle in the tibial footprint and clasps the intermediate bundle from anterior aspect (Fig. 2).

The above nomenclature differs from all descriptions in the previous studies and has been unambiguously documented in the present study. The presence and documentation of the posteromedial bundle have forced the author to alter the current nomenclature. Morphology of intermediate bundle comprising of twisted fibres has also not been documented earlier (Fig. 3).

\section{Functional significance}

Functional significance of the presence of three different bundles of different lengths cannot be undermined. In the dynamic state of the knee, joint different lengths of these bundles might resist anterior translation of tibia in different ranges of flexion. A similar mechanism is expected during different degrees of conjunct rotation of the femur on the tibia during the terminal part of the extension of the knee joint. As the femur screws home during locking of the knee joint, the spiral arrangement of Intermediate bundle and a comprehensive spiral arrangement of ACL complex as a whole (posteromedial and anterolateral bundle clasping the intermediate bundle from posterior aspect) must be playing an important role. However, this hypothesis needs further confirmation with techniques like finite element analysis.

Full extension is not possible in lower mammals [17] hence terminal extension along with conjunct rotation is peculiarly newly developed human trait and during this phase, there is an anterior translation of tibia leading to strain/ stretching of ACL and at the same time, it is accompanied by conjunct rotation for which ACL acts as the axis. The three bundles of ACL form comprehensive spirals of varying lengths, so these different bundles resist different degrees of translations and probably facilitate conjunct rotation at different angles in series by virtue of their position, spiralization and morphology in the ACL complex. Whether or not the bundles are anatomically distinct by virtue of the presence of limiting membrane but there is distinct fibre arrangement in bundles and footprints.

Technically, the ACL-R techniques have been focused on correct tunnel replacements, double-bundle repair, types of allograft to be used, etc. Few studies have explored different knee fixation angles and bundle tension protocols for double-bundle repair [13, 15, 18-20]. The present study underscores another aspect of ACL morphology i.e. differing length of bundles and their spiral arrangement. Authors are of the opinion that there is a scope of exploration of surgical techniques utilizing these anatomical facts for effective restoration and rotational stability in ACL repair.

ACL tears have been classified based on location and it has been observed that these tears are most common in the middle half of ligament. The second most common position is proximal fourth. The distal quarter is very less prone to tear [21]. Anatomically the femoral footprints are markedly smaller as compared to the tibial footprint and hence show a higher predisposition to avulsion tear. The middle half of ligament is maximally condensed and spiralled anatomically and hence expected to be strongest. However, Finite Element Analysis studies show that the same region also bears the maximum strain and hence more prone [22].

In conclusion, ACL was found to be constituted by unique spirally arranged triple bundle complex with all the bundles having differing lengths. It is hypothesized that this peculiar arrangement aids in conjunct rotation in extension and resistance to translation during different degrees of flexion of the knee joint. Finite element analysis studies are warranted to confirm the hypothesis. This knowledge may also be utilized by the surgeons to use more anatomically aligned grafts of ACL.

\section{ORCID}

Rekha Lalwani: https://orcid.org/0000-0002-3927-6069

Rohit Srivastava: https://orcid.org/0000-0003-3811-4874

Sheetal Kotgirwar: https://orcid.org/0000-0002-8321-0147 Sunita A. Athavale:

https://orcid.org/0000-0003-3003-1257

\section{Author Contributions}

Conceptualization: RL, RS. Data acquisition: RL, RS, SK, 
SAA. Data analysis or interpretation: RL, SAA. Drafting of the manuscript: RL, SK, SAA. Critical revision of the manuscript: RL, SAA. Approval of the final version of the manuscript: all authors.

\section{Conflicts of Interest}

No potential conflict of interest relevant to this article was reported.

\section{References}

1. Girgis FG, Marshall JL, Monajem A. The cruciate ligaments of the knee joint. Anatomical, functional and experimental analysis. Clin Orthop Relat Res 1975;(106):216-31.

2. Gray H, Standring S, Ellis H, Berkovitz BKB. Gray's anatomy: the anatomical basis of clinical practice. 39th ed. New York: Elsevier Churchill Livingstone; 2005. p. 1474.

3. Collins SL, Layde P, Guse CE, Schlotthauer AE, Van Valin SE. The incidence and etiology of anterior cruciate ligament injuries in patients under the age of 18 in the state of Wisconsin. Pediat Therapeut 2014;4:196.

4. Sonnery-Cottet B, Chambat P. Anatomic double bundle: a new concept in anterior cruciate ligament reconstruction using the quadriceps tendon. Arthroscopy 2006;22:1249.e1-4.

5. Kopf S, Pombo MW, Irrgang JJ, Szczodry M, Shen W, Fu FH. Size variability of the human anterior cruciate ligament insertion sites. Arthroscopy 2012;28(Suppl):e400-1.

6. Ferretti M, Alves MTS, Marcondes FB, Amaro JS, Fu FH, Cohen M. Anatomical and histological study of the anterior cruciate ligament: is there an intermediate bundle? Arthroscopy 2012;28(Suppl):e401-2.

7. Ferretti M, Doca D, Ingham SM, Cohen M, Fu FH. Bony and soft tissue landmarks of the ACL tibial insertion site: an anatomical study. Knee Surg Sports Traumatol Arthrosc 2012;20:62-8.

8. Gil YC, Park JA, Yang HJ, Lee HY. Anatomy of the femoral attachment site of the anterior cruciate ligament and the posterolateral structures related to the stability of the knee joint. Anat Cell Biol 2008;41:57-65.

9. Tan JL, Chang PC, Mitra AK, Tay BK. Anthropometry of anterior cruciate ligament in Singaporean Chinese. Ann Acad Med Singapore 1998;27:776-9.

10. Śmigielski R, Zdanowicz U, Drwięga M, Ciszek B, CiszkowskaŁysoń B, Siebold R. Ribbon like appearance of the midsub- stance fibres of the anterior cruciate ligament close to its femoral insertion site: a cadaveric study including 111 knees. Knee Surg Sports Traumatol Arthrosc 2015;23:3143-50.

11. Oka S, Schuhmacher P, Brehmer A, Traut U, Kirsch J, Siebold R. Histological analysis of the tibial anterior cruciate ligament insertion. Knee Surg Sports Traumatol Arthrosc 2016;24:74753.

12. Petersen W, Tillmann B. [Anatomy and function of the anterior cruciate ligament]. Orthopade 2002;31:710-8. German.

13. Siebold R, Ellert T, Metz S, Metz J. Femoral insertions of the anteromedial and posterolateral bundles of the anterior cruciate ligament: morphometry and arthroscopic orientation models for double-bundle bone tunnel placement--a cadaver study. Arthroscopy 2008;24:585-92.

14. Harvey A, Thomas NP, Amis AA. Fixation of the graft in reconstruction of the anterior cruciate ligament. J Bone Joint Surg Br 2005;87:593-603.

15. Siebold R, Schuhmacher P, Fernandez F, Śmigielski R, Fink C, Brehmer A, Kirsch J. Flat midsubstance of the anterior cruciate ligament with tibial "C"-shaped insertion site. Knee Surg Sports Traumatol Arthrosc 2015;23:3136-42.

16. Śmigielski R, Zdanowicz U, Drwięga M, Ciszek B, Williams A. The anatomy of the anterior cruciate ligament and its relevance to the technique of reconstruction. Bone Joint J 2016;98B:1020-6.

17. Proffen BL, McElfresh M, Fleming BC, Murray MM. A comparative anatomical study of the human knee and six animal species. Knee 2012;19:493-9.

18. Murray PJ, Alexander JW, Gold JE, Icenogle KD, Noble PC, Lowe WR. Anatomic double-bundle anterior cruciate ligament reconstruction: kinematics and knee flexion angle-graft tension relation. Arthroscopy 2010;26:202-13.

19. Smith PA, Bley JA. Anterior cruciate ligament primary repair with independent tensioning of the anteromedial and posterolateral bundles. Arthrosc Tech 2017;6:e2123-8.

20. Tanabe Y, Yasuda K, Kondo E, Kawaguchi Y, Akita K, Yagi T. Comparison of graft length changes during knee motion among 5 different anatomic single-bundle anterior cruciate ligament reconstruction approaches: a biomechanical study. Orthop J Sports Med 2019;7:2325967119834933.

21. van der List JP, Mintz DN, DiFelice GS. The location of anterior cruciate ligament tears: a prevalence study using magnetic resonance imaging. Orthop J Sports Med 2017;5:2325967117709966.

22. Park HS, Ahn C, Fung DT, Ren Y, Zhang LQ. A knee-specific finite element analysis of the human anterior cruciate ligament impingement against the femoral intercondylar notch. J Biomech 2010;43:2039-42. 